\title{
Brinzolamide/Brimonidine Fixed Combination: Simplifying Glaucoma Treatment Regimens
}

\author{
Reza Moosavi $\cdot$ Ejaz Ansari
}

Received: July 16, 2018 / Published online: October 26, 2018

(C) The Author(s) 2018

\section{ABSTRACT}

Introduction: To simplify the medical treatment of glaucoma for patients on multiple drops by introducing brinzolamide/brimonidine tartrate fixed combination (BBFC) ophthalmic suspension $1 \% / 0.2 \% \quad$ (SIMBRINZA $^{\circledR}$; Alcon Laboratories, Inc., Fort Worth, TX, USA) to the drop regimen and to establish its efficacy. To demonstrate that fixed combination (FC) therapies are associated with improvements in treatment adherence and persistence with reduced exposure to preservative-related ocular surface problems.

Methods: Retrospective study: 76 patients were identified as taking BBFC following a switch in treatment regimen. Intraocular pressure (IOP) prior to and 2-17.5 months (average 5.4 months) after the introduction of BBFC was measured. The change in the average number of bottles used per eye was recorded. The rate of adverse effects (AEs) of BBFC was recorded. A two-tailed paired sample $t$ test was used to

Enhanced digital features To view enhanced digital features for this article go to https://doi.org/10.6084/ m9.figshare.7172015.

R. Moosavi $(\varangle) \cdot$ E. Ansari

Eye Ear and Mouth Unit, Maidstone and Tunbridge Wells NHS Trust, Maidstone Hospital, Maidstone, UK

e-mail: rezamoosavi@nhs.net compare IOP prior to and after the introduction of BBFC for each eye.

Results: Mean change in IOP after BBFC introduction BBFC: $-2.76 \mathrm{mmHg} \quad(p<0.0001)$. BBFC intolerance: $13 \%$. On average there was a 0.24 reduction in the number of bottles of IOPlowering medication used per eye $(p<0.0064)$. Conclusion: A switch to BBFC in the drop regimen is associated with a significant drop in IOP with reduced drop burden. Instead of a third IOP-lowering medication and bottle, a practitioner should consider using BBFC + prostaglandin analogue/FC drop for effective IOP control, reduced drop burden, reduced preservative load and increased likelihood of adherence. This study promotes the concept that any treatment should principally be assessed from the patients' perspective and quality of life.

Keywords: Brinzolamide-brimonidine fixed combination; Ophthalmology; Simbrinza

\section{INTRODUCTION}

Glaucoma remains the major cause of irreversible vision loss worldwide. In 2010 it was estimated to have caused over 8 million cases of blindness, and by 2020, 79 million people worldwide will have glaucoma [1]. Glaucoma is characterised in most cases by elevated intraocular pressure (IOP), with progressive 
optic neuropathy being the main diagnostic criterion, and corresponding visual field loss $[2,3]$. Currently, IOP remains the only modifiable risk factor [4].

Many topical IOP-lowering agents with different mechanisms of action are available, e.g., $\beta$-blockers, prostaglandin analogues (PGAs), carbonic anhydrase inhibitors (CAIs), $\alpha 2$ adrenergic agonists and parasympathomimetic agents, e.g., pilocarpine [5-7]. These medications reduce IOP by decreasing aqueous production [5], increasing aqueous outflow [5-10] or both. In contrast, PGAs work by increasing uveoscleral and trabecular meshwork outflow facility $[8,11]$, and $\alpha 2$-adrenergic agonists reduce aqueous production and augment aqueous outflow through the uveoscleral pathway [12]. Recommended first-line treatment for glaucoma is using a single IOP-lowering medication [7-13]. However, one study showed that $40 \%$ of patients need polypharmacy to reach and maintain their target IOP, and an additional 9\% needed more than three medications [14]. The use of multiple drops in a chronic and often asymptomatic condition can lead to lack of persistence and non-adherence, which may ultimately decrease drug effectiveness [15]. Studies demonstrate that approximately $50 \%$ of patients have been found not to be adherent to their medication over $75 \%$ of the time [16]. Medical treatment regimens requiring separate administration of several topical agents tend to have lower persistence $[17,18]$.

A method of improving adherence and persistence with glaucoma drops is to use fixedcombination medications, which allow instillation of two medications in a single solution. Fixed-combination medications reduce the number of medication bottles, reduce costs and simplify the dosing regimen, all of which may increase persistence [17-19] and adherence $[17,18]$. A prospective trial has shown that switching from concomitant administration of multiple separate medications to a fixed-combination therapy increases patient adherence [20]. By using fixed combinations, tolerability may be improved over the use of multiple agents by reducing the cumulative exposure to irritating preservatives [21]; therefore, the reduced ocular symptoms associated with fixed- combination medications may improve overall adherence [22].

Whilst most combination preparations contain timolol, which is a beta-blocker, there are several potential side effects of beta-blockers that can limit patient adherence and persistence with these drugs; furthermore they are contraindicated in patients with certain medical conditions, such as asthma, severe chronic obstructive pulmonary disease, sinus bradycardia, impotence, depression, confusion and memory loss [23].

Brinzolamide $1 \%$-Brimonidine $0.2 \%$ fixed combination/BBFC (SIMBRINZA ${ }^{\circledR}$; Alcon Laboratories Inc, Fort Worth, TX, USA) remains the only combination preparation for glaucoma and ocular hypertension that does not contain a beta-blocker. It is a sterile, preserved ophthalmic suspension formulation with a safety profile similar to its individual components. BBFC is approved for three-times-daily dosing in the USA and two-times-daily dosing in Europe.

We estimated that $>20 \%$ of our glaucoma clinic population was on three or more separate drop bottles at one time. Our aim was to study the effect of simplifying glaucoma treatment regimens in patients taking multiple bottles of glaucoma drops $(>2)$ by maximising the combination preparations prescribed, thereby reducing the numbers of bottles used with the aim of improving adherence and IOP control. BBFC was used in the new treatment regimens. The purpose of this study is to assess the efficacy of the new treatment regimen and tolerability of the same over at least a 2-month period following a switch or simplification of therapy.

\section{METHODS}

The study was exempt from requiring Institutional Review Board approval as it involved the assessment of retrospective and de-identified data. No ethical issues were identified.

\section{Patient Identification}

This study was conducted through retrospective case note review for patients attending the glaucoma clinics at the Eye Ear and Mouth Unit, 
Maidstone and Tunbridge Wells NHS Trust, between October 2014 and July 2016. We collected and reviewed the medical records of patients whose topical intraocular pressure (IOP)-lowering regimen had been changed to include the use of BBFC.

As a result of the recent introduction of an electronic patient record at this NHS Trust, some patients' records were in the form of paper notes and others in the form of an electronic patient record.

\section{Inclusion Criteria}

Eligible patients were aged $\geq 18$ years, diagnosed with open angle glaucoma (OAG), including pseudoexfoliation, pigmentary glaucoma or ocular hypertension. These patients had used three or more different bottles of drops before the switch to a regimen including BBFC.

\section{Exclusion Criteria}

Patients with unobtainable or incomplete records were excluded from further analysis. Patients identified as taking BBFC drops with a follow-up period $<2$ months were excluded from data analysis.

\section{Data Collection and Analysis: Outcome Criteria}

For eligible patients with complete records who met all the inclusion or exclusion criteria, the following outcome criteria were obtained for each eye:

1. The IOP \{as measured by Goldmann applanation tonometry (GAT)] prior to and most recently after the introduction of BBFC.

2. The time interval between the change in regimen and the most recent IOP reading.

3. The total number of IOP-lowering drop bottles used prior and subsequent to the change in regimen.

4. The number of patients unable to tolerate or persist with BBFC as a result of side effects was recorded.

In this study, the treatment regimen was changed as follows. For example, if a patient was taking latanoprost + timolol + brimonidine + brinzolamide (4 separate bottles), the treatment regimen was rationalised to lataoprost/timolol $0.5 \%+$ BBFC, thereby halving the bottle burden, but maintaining four active components in just two bottles. This has now become possible with the introduction of BBFC to the glaucoma pharmacopoeia.

\section{Statistical Methods}

Statistical analysis was performed using a twotailed paired sample $t$ test to compare IOP prior to and after the introduction of BBFC for each eye involved.

A two-tailed paired sample $t$ test was used to compare the average number of IOP-lowering drop bottles prior to and after the introduction of BBFC for each eye involved.

\section{RESULTS}

A total of 85 patients were identified in the glaucoma clinics at Maidstone and Tunbridge Wells NHS Trust as taking BBFC following a switch in treatment regimen using the identification method outlined above. Patients were 18-80 years of age.

Nine patients' notes were incomplete or unobtainable.

Ten of the 76 remaining patients were unable to continue with BBFC because of intolerance (dropout rate 13\%). Sixty-six of 76 (87\%) patients showed continued adherence at most recent follow-up.

Six of 76 additional patients were excluded from IOP analysis; they were identified as having started BBFC without side effects, but longer than 2 months' follow-up IOP results were unavailable.

After these exclusions, 85 eyes of 60 patients subsequently underwent data analysis.

For each eye, IOP measurements prior to and 2-17.5 months (mean follow-up 5.4 months) after the introduction of BBFC were recorded.

The change in IOP after the introduction of BBFC for each eye is summarised in Fig. 1. The mean change in IOP after the introduction of BBFC was $-2.76 \mathrm{mmHg}$ : two-tailed paired 


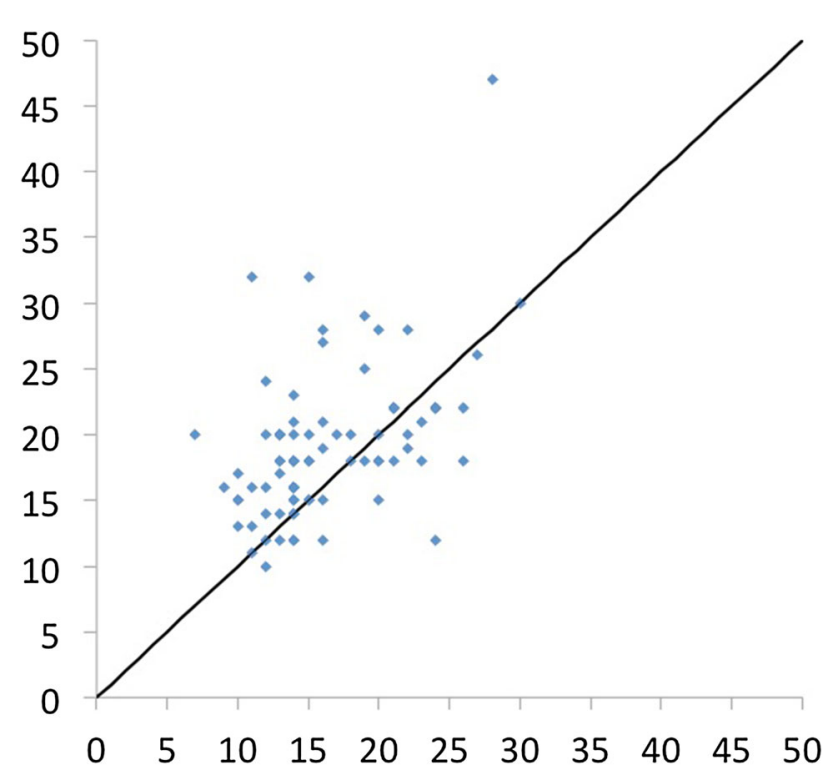

Fig. 1 Chart: IOP change $(\mathrm{mmHg})$ pre $(y$-axis) and post ( $x$-axis) addition of BBFC

sample $t$ test; $p<0.0001$ [95\% CI for mean difference (1.59-3.94) intermediate values used in calculations: $t=4.6861 ; d f=84$; standard error of difference $=0.590$ ] (see Table 1$)$.

On average there was a 0.24 reduction in the number of bottles of IOP-lowering medication used per eye after the introduction of BBFC to the drop regimen: two-tailed paired sample $t$ test; $p<0.0064$ [95\% CI for mean difference (0.07-0.42) intermediate values used in calculations: $t=2.7931 ; d f=84$; standard error of difference $=0.087]$.

\section{DISCUSSION}

In this study we found that reducing the bottle burden and therefore drop burden for patients

Table 1 IOP analysis results (see Fig. 1)

\begin{tabular}{ccccc}
\hline $\boldsymbol{N}$ & $\begin{array}{l}\text { Pre- } \\
\text { change } \\
\text { IOP }\end{array}$ & $\begin{array}{l}\text { Post- } \\
\text { change } \\
\text { IOP }\end{array}$ & $\begin{array}{l}\text { Mean } \\
\text { change } \\
\text { in IOP }\end{array}$ & $\begin{array}{l}\text { Mean change in } \\
\text { number of } \\
\text { bottles used per } \\
\text { eye }\end{array}$ \\
\hline 85 & Mean & Mean & Mean & Mean -0.24 \\
eyes & 19.0 & 16.2 & change & $(0.24$ \\
& $(\mathrm{SD}$ & $(\mathrm{SD}$ & in IOP & reduction $)$ \\
& $5.69)$ & $4.84)$ & -2.76 & \\
\hline
\end{tabular}

with OAG and OHT not only maintained IOP control but also actually improved it. This could be related to improved compliance generally, since after the switch, a maximum of two bottles were used instead of three or more. In addition, most patients were able to tolerate the new treatment regimen that included BBFC.

The improved IOP control could also be due to the BBFC preparation. In a recent randomised clinical trial (RCT), addition of BBFC to PGA monotherapy realised a mean diurnal IOP at 6 weeks that was significantly lower compared with vehicle + PGA [(least square mean \pm SE diurnal IOP was $17.1 \pm 0.4 \mathrm{mmHg}$ with $\mathrm{BBFC}+\mathrm{PGA}$ (95\% CI 16.3-17.8 mmHg) vs. $20.5 \pm 0.4 \mathrm{mmHg}$ with vehicle $+\mathrm{AA}(95 \%$ CI 19.8-21.2 mmHg)] [24]. The additional IOP reduction with BBFC (mean diurnal IOP reduction of $25 \%$ at week 6 ) was significantly greater than that with the addition of its individual components in a previous study [25].

In another study, fixed-combination brinzolamide/timolol or brimonidine/timolol added to travoprost reduced IOP from travoprosttreated baseline $(20.1 \mathrm{mmHg})$ by 14 and $10 \%$, respectively, after 3 months [26]. This suggests that BBFC added to a PGA may produce IOP reductions equivalent to those in previous studies of a three-agent treatment regimen including a $\beta$-blocker. 
Our study was different in that it rationalised and streamlined medical treatment by reducing the drop burden (subtraction method) rather than adding drops. However, the above studies confirmed the efficacy of BBFC in combination with PGA monotherapy, which is in effect what our study patients were taking post-switch (either $\mathrm{BBFC}+\mathrm{PGA}$ monotherapy or BBFC + PGA/timolol 0.5\%). Our study confirmed that it is safe to rationalise treatment in this way since IOP control improved postswitch.

The mean reduction in IOP in our study $(2.76 \mathrm{mmHg})$ was significant and beneficial in more than one way. For example, results of the Early Manifest Glaucoma Trial (EMGT) showed that the risk of progression decreased by $10 \%$ with each $1-\mathrm{mmHg}$ decrease in mean IOP [27]. In our study, the mean IOP decrease from baseline was $>2.5 \mathrm{mmHg}$ at an average of 5.4 months follow-up. In a meta-analysis of four studies $(n=822)$, mean IOP was a significant risk factor for progression [28]. Progression over 5 years occurred in $>50 \%$ with mean IOP $>20 \mathrm{mmHg}$ compared with $\leq 18 \%$ who had mean IOP of $13-17 \mathrm{mmHg}$ [27]. In our study, mean IOP was decreased from 19 (SD 5.69) $\mathrm{mmHg}$ at baseline to 16 (SD 4.84) $\mathrm{mmHg}$, i.e., on average $<17 \mathrm{mmHg}$, implying a reduced risk of disease progression in the long term.

The treatment regimen including BBFC was well tolerated. Thirteen per cent of patients discontinued BBFC because of adverse effects including allergic conjunctivitis, eyelid inflammation and oedema, ocular irritation and/or pain, iridocyclitis, hyperaemia, increased lacrimation and blurred vision. Patients who had been taking and tolerating brimonidine $0.2 \%$ also tolerated BBFC subsequently.

The limitations of this study are generally those of a retrospective study. As such there was no control group and no wash-out period established between drop changes. Also, the range of follow-up was wide at 2-17.5 months (mean 5.4). However, even at 2-month followup the pre-switch medications would have washed out giving a true indication of the postswitch IOP and tolerability. Follow-up for a longer period of time, e.g., a mean of
12 months, would help to establish the longer term efficacy and tolerability of BBFC. A future prospective randomised trial with a control group would be appropriate with diurnal IOP measurements in a masked fashion as well as quality of life assessments. Measurements of the ocular surface condition such as the ocular surface disease index, osmolarity, tear break-up time and conjunctival/corneal staining would also be valuable in such a study to examine the effect of a reduced benzalkonium chloride (BAK) load to the ocular surface [29].

\section{CONCLUSIONS}

In conclusion, rationalising topical treatment with the inclusion of the new combination preparation BBFC (subtraction method) was effective and safe. It led to a mean IOP reduction of $>2.5 \mathrm{mmHg}$ at an average of 5 months' follow-up. The new treatment regimen was well tolerated in $87 \%$ of cases. There were no major adverse events beyond the known side effects of the individual components used in the treatment regimen. Therefore, clinicians are advised to try to minimise topical treatment at every opportunity and to avoid polypharmacy by prescribing more combination preparations including BBFC where possible.

\section{ACKNOWLEDGEMENTS}

We thank the study participants.

Funding. No funding or sponsorship was received for this study or publication of this article. The article processing charges were funded by the authors.

Authorship. All named authors meet the International Committee of Medical Journal Editors (ICMJE) criteria for authorship for this article, take responsibility for the integrity of the work as a whole, and have given their approval for this version to be published.

Disclosures. Reza Moosavi has nothing to disclose. Ejaz Ansari has received fellowship 
grants, research funding and speaker fees from Allergan, educational grant and speaker fees from Thea and speaker fees from Alcon.

Compliance with Ethics Guidelines. The study was exempt from requiring Institutional Review Board approval as it involved the assessment of retrospective and de-identified data.

Open Access. This article is distributed under the terms of the Creative Commons Attribution-NonCommercial 4.0 International License (http://creativecommons.org/licenses/ by-nc/4.0/), which permits any noncommercial use, distribution, and reproduction in any medium, provided you give appropriate credit to the original author(s) and the source, provide a link to the Creative Commons license, and indicate if changes were made.

\section{REFERENCES}

1. Quigley HA, Broman AT. The number of people with glaucoma worldwide in 2010 and 2020. Br J Ophthalmol. 2006;90:262-7.

2. Casson RJ, Chidlow G, Wood JP, Crowston JG, Goldberg I. Definition of glaucoma: clinical and experimental concepts. Clin Exp Ophthalmol. 2012;40:341-9.

3. Peters D, Bengtsson B, Heijl A. Factors associated with lifetime risk of open-angle glaucoma blindness. Acta Ophthalmol. 2014;92(5):421-5.

4. The AGIS Investigators. The Advanced Glaucoma Intervention Study (AGIS): 7. The relationship between control of intraocular pressure and visual field deterioration. Am J Ophthalmol. 2000;130:429-40.

5. Webers CA, Beckers HJ, Nuijts RM, Schouten JS. Pharmacological management of primary openangle glaucoma: second-line options and beyond. Drugs Aging. 2008;25:729-59.

6. American Academy of Ophthalmology Glaucoma Panel. Preferred Practice Pattern ${ }^{\circledR}$ Guidelines. Primary open-angle glaucoma. San Francisco: American Academy of Ophthalmology; 2010.

7. Realini T. A history of glaucoma pharmacology. Optom Vis Sci. 2011;88:36-8.
8. Nguyen QH. The role of prostaglandin analogues in the treatment of glaucoma in the 21st century. Int Ophthalmol Clin. 2004;44:15-27.

9. Watanabe K, Chiou GC. Action mechanism of timolol to lower the intraocular pressure in rabbits. Ophthalmic Res. 1983;15:160-7.

10. Mincione F, Scozzafava A, Supuran CT. The development of topically acting carbonic anhydrase inhibitors as anti-glaucoma agents. Curr Top Med Chem. 2007;7:849-54.

11. Toris CB, Gabelt BT, Kaufman PL. Update on the mechanism of action of topical prostaglandins for intraocular pressure reduction. Surv Ophthalmol. 2008;53(Suppl 1):S107-20.

12. Arthur S, Cantor LB. Update on the role of alphaagonists in glaucoma management. Exp Eye Res. 2011;93:271-83.

13. European Glaucoma Society. Terminology and guidelines for glaucoma, 3rd ed. Bern: European Glaucoma Society. http://www.eugs.org/eng/EGS guidelines.asp. Accessed 24 Jan 2014.

14. Kass MA, Heuer DK, Higginbotham EJ, et al. The Ocular Hypertension Treatment Study: a randomized trial determines that topical ocular hypotensive medication delays or prevents the onset of primary open-angle glaucoma. Arch Ophthalmol. 2002;120:701-13.

15. Vorwerk C, Thelen U, Buchholz P, Kimmich F. Treatment of glaucoma patients with insufficient intraocular pressure control: a survey of German ophthalmologists in private practice. Curr Med Res Opin. 2008;24:1295-301.

16. Okeke CO, Quigley HA, Jampel HD, Ying GS, Plyler RJ, Jiang Y, et al. Adherence with topical glaucoma medication monitored electronically: the travatan dosing aid study. Ophthalmology. 2009;116:191-9.

17. Higginbotham EJ, Hansen J, Davis EJ, Walt JG, Guckian A. Glaucoma medication persistence with a fixed combination versus multiple bottles. Curr Med Res Opin. 2009;25:2543-7.

18. Schwartz G, Burk C, Bennett T, Patel VD. Adherence and persistence with glaucoma therapy: brimonidine/timolol versus dorzolamide/timolol and various two-bottle combinations. J Clin Exp Ophthalmol. 2012;3:1-6.

19. Hommer A, Thygesen J, Ferreras A, et al. A European perspective on costs and cost effectiveness of ophthalmic combinations in the treatment of open-angle glaucoma. Eur J Ophthalmol. 2008;18:778-86. 
20. Inoue $\mathrm{K}$, Okayama R, Higa R, Wakakura M, Tomita G. Ocular hypotensive effects and safety over 3 months of switching from an unfixed combination to latanoprost $0.005 \% /$ timolol maleate $0.5 \%$ fixed combination. J Ocul Pharmacol Ther. 2011;27:581-7.

21. Kitazawa Y, Smith P, Sasaki N, Kotake S, Bae K, Iwamoto Y. Travoprost 0.004\%/timolol 0.5\%-fixed combination with and without benzalkonium chloride: a prospective, randomized, doubledmasked comparison of safety and efficacy. Eye (Lond). 2011;25:1161-9.

22. Holló G, Topouzis F, Fechtner RD. Fixed-combination intraocular pressure-lowering therapy for glaucoma and ocular hypertension: advantages in clinical practice. Expert Opin Pharmacother. 2014;15:1737-47.

23. Taniguchi T, Kitazawa Y. The potential systemic effect of topically applied $\beta$-blockers in glaucoma therapy. Curr Opin Ophthalmol. 1997;8:55-8.

24. Fechtner RD, Myers JS, Hubatsch DA, Budenz DL, DuBiner HB. Ocular hypotensive effect of fixedcombination brinzolamide/brimonidine adjunctive to a prostaglandin analog: a randomized clinical trial. Eye. 2016;30:1343-50.
25. Whitson JT, Realini T, Nguyen QH, McMenemy MG, Goode SM. Six-month results from a phase III randomized trial of fixed-combination brinzolamide $1 \%+$ brimonidine $0.2 \%$ versus brinzolamide or brimonidine monotherapy in glaucoma or ocular hypertension. Clin Ophthalmol. 2013;7:1053-60.

26. Konstas AG, Holló G, Haidich AB, et al. Comparison of 24-h intraocular pressure reduction obtained with brinzolamide/timolol or brimonidine/timolol fixed-combination adjunctive to travoprost therapy. J Ocul Pharmacol Ther. 2013;29:652-7.

27. Heijl A, Leske MC, Bengtsson B, Hyman L, Bengtsson B, Hussein M, Early Manifest Glaucoma Trial Group. Reduction of intraocular pressure and glaucoma progression: results from the Early Manifest Glaucoma Trial. Arch Ophthalmol. 2002;120:1268-79.

28. Stewart WC, Kolker AE, Sharpe ED, et al. Long-term progression at individual mean intraocular pressure levels in primary open-angle and exfoliative glaucoma. Eur J Ophthalmol. 2008;18:765-70.

29. Actis AG, Rolle T. Ocular surface alterations and topical antiglaucomatous therapy: a review. Open Ophthalmol J. 2014;8:67-72. 